\title{
PCR detection and sequencing of parasite ITS-rDNA gene from reservoirs host of zoonotic cutaneous leishmaniasis in central Iran
}

Parviz Parvizi • Ghasem Moradi • Ghasem Akbari •

Mahin Farahmand • Paul D. Ready • Norair Piazak •

Mehdi Assmar • Aref Amirkhani

Published online: 16 October 2008

(C) Springer-Verlag 2008

\section{Erratum to: Parasitol Res}

DOI 10.1007/s00436-008-1124-z

The original version of this article unfortunately contained a mistake. The presentation of Table 3 was incorrect. The corrected table is given below.

\footnotetext{
The online version of the original article can be found at http://dx.doi. org/10.1007/s00436-008-1124-z.

P. Parvizi $(\bowtie) \cdot$ G. Moradi $\cdot$ M. Farahmand $\cdot$ N. Piazak

M. Assmar

Department of Parasitology, Pasteur Institute of Iran,

69 Pasteur Ave.,

Tehran, Iran

e-mail: parp@pasteur.ac.ir

G. Moradi

Department of Microbiology, Azad Islamic University of Zanjan,

Zanjan, Iran

G. Akbari

Centre of Health Service,

Natanz, Isfahan Province, Iran

P. D. Ready

Department of Entomology, Natural History Museum,

London, UK

A. Amirkhani

Department of Epidemilogy, Pasteur Institute of Iran,

Tehran, Iran
} 
Table 3 Leishmania species and isolates analysed of their ITS-rDNA fragment sequences and alignment of all variant nucleotide positions of Leishmania infections from rodents in Natanz, Isfahan province and GenBank

\begin{tabular}{|c|c|c|c|}
\hline \multirow{2}{*}{\multicolumn{2}{|c|}{ GenBank }} & \multirow[b]{2}{*}{ Leishmania } & \multirow{2}{*}{$\begin{array}{c}\text { variant character position } \\
000000000000000000000111111112222222333\end{array}$} \\
\hline & & & \\
\hline Accession & & Species & 122223333344458889999022457881122347333 \\
\hline No. & Origin & & 913791256867960351234736612370635807346 \\
\hline R16 (EU871037 & 7) Iran & L. tropica & AGCAATA-CTAGCCCTAGAAGCCACCT-TG---TTTGCG \\
\hline AJ300485 & Tunisia & L. tropica & AGCAAATTTC-A-CCTGGAAGCGACCT-TG---TTAGCG \\
\hline AJ000302 & Namibia & L. tropica & AGCA-ATC-TAG-CCTAGAAGCCACCT-TC---TTAGCG \\
\hline AJ 000301 & Kenya & L. tropica & AGCAA-TT-C-A-CCGAGAAGCGACCT-TG-- - TTAGCG \\
\hline AJ 000310 & İU.S.S.R̂̀ & L. tropica & AGTTCATT-T-ATTCTAAAAAAGGCCTAGGGAGCCACGG \\
\hline $\mathrm{AJ} 300482$ & Kenya & L. major & AGTTCATT-T-ATTCTAGAAAAGGCCTAGGGAGCCAGCG \\
\hline EF413077 & Iran & L. major & AGTTCATT-T-ATTCTAAAAGAGGCCTAGGGAGCCAGCG \\
\hline EF413078 & Iran & L. major & AGTTCATT-T-ATTCTAAAAAAGGCCTGGGGAGCCAGCG \\
\hline R05 (EF413075 & 5) Iran & L. major & AGTTCATT-T-ATTCTAAAAAAGGCCTAGGGAGCCAGCG \\
\hline AJ272381 & Turkmenistan & L. turanica & CGCTAATT-TTA-CCTAGAAAAGACCGATGGTTCCAGCG \\
\hline AJ272380 & Mongolia & L. turanica & CGCTAATT-TTA-CCTAGAAAAGACCGATGGTTCCAGCG \\
\hline $\mathrm{AJ} 300486$ & Uzbekistan & L. gerbilli & AGCTAATT-TTATCCTAGCGAAGAGTGATGGTTCCAGCC \\
\hline AJ634354 & Italy & L. infantum & AACG-- - T-T-G-CGTGAAAAAGACCTATGT-- -TAGCG \\
\hline AJ634370 & Sudan & L. infantum & AACGA- - T-T-G-CGTGAAAAAGACCTATGT-- - TAGCG \\
\hline AJ634375 & India & L. donovani & AACGA- - T-T-G-CGTGAAAAAGACCTATGT- - TAGCG \\
\hline AJ634360 & Ethiopia & L. donovani & AACG-- - T-T-G-CGTGAAAAAGACCTATGT- - TAGCG \\
\hline AJ634358 & Sudan & L. archibaldi & AACG-- - T-T-G-CGTGAAAAAGACCTATGT- - TAGCG \\
\hline AJ634367 & Ethiopia & L. archibaldi & AACGAATT-T-G-CGTGAAAAAGACCTATGT-- - TAGCG \\
\hline
\end{tabular}

